graph does not share the errors of the human observer to which I have called your attention, it tails in that critical and expectant attention which only the human observer can exercise, and which, if it sometimes leads him into error, yet makes him more efficient than any. mechanical recording apparatus.

You cannot afford to ignore any detail, you have to be on the alert for every trifle, you have to be quick to interpret anything that is new: I may, therefore, hope to be pardoned for reminding you that these qualities may, be actually deadened by a too rigid adherence to the methods by which we identify and study what is known. I sometimes feel that many scientific workers and teachers. forgot that advance is made not so much by stadjing the known as by seeking the unknown, and that it is the differences, ther:novelties, the eccentricities if you like, which characterize the new thing, that should excite our expectations. The object of studying a known form with minute precision is not only that we may recognize it again when we see it, but also that we mas distinguish from it the new form when it presents itself, however closely it may resemble the known; and, unless. this object is constantly kept in view, we may fall into the error of either noticing the resemblances more than the differences, or ot attaching undue importance to differences that are inessential bat easily discernible.

I feel that it is somewhat presumptrous on my part to make such suggestions in regard to a science of which. I know nothing; but I am, so. fully conscious of their validity. in the history of mineralogy a science with which I am familiar, and it is so probable that the ways of discovery and of erroc are the same in all the sciences, that I have little doubt that what I say is also applicable to bacteriology and to the subject of tropical medicine.

Those who are stadents here come, I understand, with the object of acquainting themselves with the best. and nowest mothods of research, and their further studies are pursued in distent paxts of the world, where they have not the advantages of the elaborate equipment and excellent opportunities. which are afforded them in this school. Many will be doing pioneer work undèr difficult and novel conditions, in whioh, far more than in a well-equipped hospital or school, new problems are to be expected, and the faculty of observation is on its trial.

In all scientific work we should be on our guard; not let our knowledge get the better of us; but be on the alert for something new even in that which is most familiar, and in the routine study of that which is best known.

\section{Ant Adidregs}

\section{CERTAIN INTRACRANIAL DISEASES ASSO- CIATED WITH NASAL, AURAL, AND LARYNGEAL SYIIPTOMS.}

Dhliverbe at the Centrat: London: Throat and Ear Hospitax on Ootober 17Th, 1910.

BY

PURVES STEWART, M.A., M.D., F.R.C.P., CONSOLTING. PHYSIOIAN TO THE. HOSPITAL: PHYSICIAN TO THE WEBTMINSTER HOSPIT HOSPITAI TOR NERTOUS THE WES.

My first duty is to offer my sincere thanks for the honour that has been done me in inviting me to address you to-day, and to express the pleasure with whioh I accepted that invitation. At the same time I cannot help recog. nizing that my. task is difficalt, not only in viow of the distinction of the orators who have preceded me in former years, but alse from the character of the andience that I now see before me.

Of late years the study of disesses of the throat, nose, and ear has made great advances-advances in which many of yourselves have played an important part. To the average practitioner of medi. cine the special domains of laryngo. logy, rhinology, and otology may well seem a labyrinth to which only the chosen tow can hope to possess the clue. My object to.day, however, is not to illustrate the im. portance of rhinology, otology, and

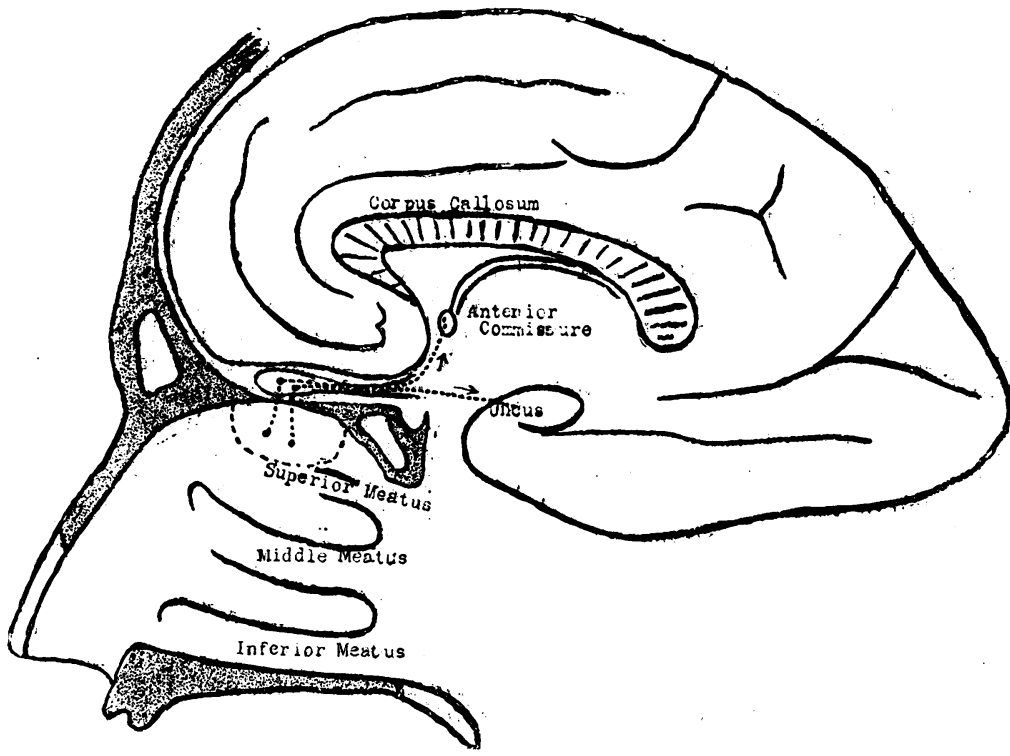

Fig. 1.-Diagram of connexions of olfactory nerves.

In a hospital such as this you have to deal usually with patients who come to you complaining of symptoms which they themselves associate with the ear, the nose, or the throat. A certain, small proportion of these cases is associated with intracranial complications-as, for example, temporal or cerebellar abscess, meningitis, lateral sinus thrombosis, etc., secondary to ear suppuration; frontal brain abscess or cavernous sinus thrombosis, secondary to. nasal infection, and so on. With cases of this sort I do not propose to deal. I propose to direct your attention to some primarily intracranial diseases, where the aural, nasal, or laryngeal, symptoms only transpire incidentally, as it were, in the course of the malady. and where, neverthelese, the recognition of symptoms connected with the special domains of rhinology, otology, and larpngology is of the utmost importance for a correct diagnosis.

I trust you will forgive me if I recall to your memory a few of the more important points in the anatomy of the nerve tracts which are in special relation with the nose and ear.

The olfactory nerves ramify in the mucasa of the upper and baok part of the nasal septum, and over the surface of the upper turbinal body in the outer wall of each. nasal fossa. They perforate the cribriform plate of the ethmoid bone. Piercing the dura mater, they reach the superjacent olfactory bulb of the corresponding side. The olfactory nerves are enclosed in lymphatic spaces which are directly continuons with the subarachnoid space, so that the lymphatics of the upper nasel mucose cen readily be injected from the cranial cevity by mesns of substances such as Prussian blue intro. duced into the cere. bral subarachnoid space. Notwith. standing this free communication, it is

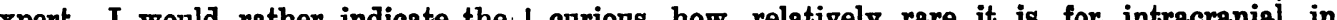
value of an elementary knowledge of these special branches to the general physician or surgeon, and more especially to the neurologist. curious how relatively rare it is for intracranial in-
fections to arise from nasal as compared with aural conditions.

From the olfactory bulb the olfactory tract or peduncle 
leads beckwatis to the anterior perforeted mace, or olfactory tubercle, on the under surface of the frontal lobe. There it bifurcates into a mesial and a lateral root. Most of the fibres in the lateral root ran to the olfactory cortex at theitip of the hippocampal region (uncinate gyrus). Other fibres, in the mesial root, run to the anterior commissure, where they cross the middle line and reach the uncus of the opposite side. We note that the olfactory path does not traverse the internal capsule, and that, therefore, in the ordinary type of organic hemiplegia, smell is unaffected.

The course of the gustatory path is still a subject of dispate. Most of us believe that impressions of taiste onter the brain partly through the sensory root of the trigeminal nerve, partly through the nervas intermedius or sen. sory root of the facial. and partly through the glosso-pharyngeal nerve. We know also that the cortical centre for taste is situated towards the tip of the temporal lobe, close to the centre for smell. The exact course of the intra. corebral ig uistatory path is in dispute, but it is probablo that it does not traverse the internal capsule. Not fur from the centres for smell and taste in the temporal lobe there appear to be cortical centres for hunger and thirst, which are sometimes of considerable dia. gnostic importance, as wo shall presently see.

The auditory epparatus has to deal with two distinct sets of impressions. First, cochlear or acoustic im. pressions, and secondly, vestibular impressions of equilibration from the semicircular canals. These two sots of impulses have different paths within the brain.

The acoustic path from the cochlea passes along the cochlear nerve. This bifurcates to embrace the restiform body botween its arms. Some of its fibres pass internally and go to the ventral auditory nucleas in the upper part of the medulla: others pass along the outer side of the restiform body and go to the dorsal auditory nucleng. From these two medullary nuclei the fibres of the central sooustic path lead upwards towawds the higher auditor contres. Mast (but not sill) of them decusinte to the opposite side and ascend in the latenal fillet, thich conducts them to the posterior corpus quadrigeminum and the mesial genioulate boaly. From thene nnolei now acoustic fibres traverwe the rablenticular region of the internal espsule (the auditory fibres being perterfor to the Abwes for ordinary cutancous menstions), and tifimatel

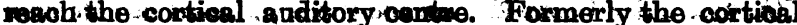

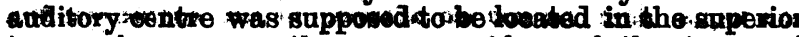
temporal gyeus : On the convex sullave of the tempowa Iobe. Recently, howerer, we have eome to rocognize that enother important part of the auditory centre is in the anterior transverse temporal convolution of Heschl for Fleohsig's anditory gyras), situated on the upper or Sylvien surface of the temporal lobe at the bottom of the Sylvian fosss, elose behind the insula. Heschl's gyrus is directly continuous with the superior temporel gyrus. Fach cortical centre receives anditory messages from both ears (although more extensively from the ear of the contra. lateral side), so that a lesion of one temporal lobe does not canse desiness. Finally, in right.handed people there is a specially differentiated portion of the loft euditory centre, where memories of word-sounds are stored up. This is of special importance in temporal abscess-a not uncommon complication of otitis media.

The vestibular path from the semicircular canals penetrates the medulla and reaches the vestibular nucleus. Thence it passes to Deiters's nucleus of the same side, from which a new path leads up to tho vermis or midale lobe of the cerebellum.

Tet me now relate a few csses of intra. cranial lesions in which the nasal, cochlear, vestibular, or laryngeal symptoms were of speeial dia. gnostic value.

Case I.-Tumour in Re. gion of Optic Chiasma.

A widow of 61 noticed gradual failure of vision some thirty years previously, so that the right eye became totally blind. Some four or five years ago she noticed impairment of vision in the left eye. Two years ago she began to have attacks of overwhelming drowsiness and used to fall asleep at meals fall asleep at meals. Her appetite became voracious. Giddinoss and an unsteady gait developed, and she nsed to run into people in the street, apparently owing to hemianopia in the eye which was still able to see. Causeless vomiting supervened. Vertical headaches appeared. The drowsiness pontinued and when continued, and when she came to us at Westminster Hospital she slept ously, requiring to be ously, requiring to

wakened for meals. On examination, she could smell aromatic left nostril, less in the right. There was optic atrophy in both eyes, with total blindness of the right eye and temporal hemianopia of the left-signs pathognomonic of a lesion of the optic ohiowa. reacted sluggishly. All the other cranial nerves were normal. There was no dotheness. There was'alight weaknens of the left lower fwoe, cerebral in type. 'There was no paralysis or aturia' bef any Hmib; and the refexes, deeptand superfoial, whowed nơ abnormility

She was mentally aull, slept almost onnstantly, but retaired a remutkubly keen appetite. In spite of this, however, 'when being fed, the would thil osteop botween'mouthfuls.

The prolonged course of the lasewse, the headache; romiting, and optic atrophy, pointed to an intracrenial growth. The blindness of the right ere and temporal hemianopia of the: left, were charactriatic of a lesion 'of the optic ohiasma, more on

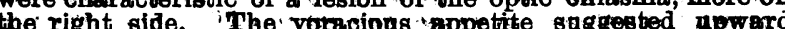
the righ slde. The voracious elight froial hemiplegia suggested that the growth pressed 
against the motor path in the right hemisphere. An attempt was made by my colleague, Mr. W. Turner, to reach the growth from the right frontal region, but two days after the first stage of the operation the patient died from heart failure.

At the autopsy our diagnosis was amply confirmed, for we found a large tumour in which were embedded the optic chiasma and right carotid artery. It compressed the third ventricle, and pressed upwards against the crura cerebri and temporal lobes, especially on the right side. This growth seemed to have
arisen from the infundibulum, the anterior lobe of the pitaitary body being normal in size, situated within the sella turcica.

This case is of interest to us, as exemplifying the importance close proximity to the centres for smell and taste.

CASE II.-Right-sided Extracerebellar Tumour arising from Auditory Nerve.

A married woman, aged 32, complained of unsteadiness in walking for about two years. For twelve months she had had occasional cervico-occipital headaches, with nausea and vomiting independent of food. She had never had an attack of unconsciousness. Within the last five months the unsteadiness in walking had rapidly increased, and she occasionally fell without unconsciousnuss, noticing at these times a transient without unconsciousness, noticing at these times a transient
weakness of the right arm. For three months she had been weakness of the right
blind in the right eye.

On examination, she was an intelligent woman with normal speech and articulation. There was intense donble optic neuritis, with 3 dioptres of swelling in the right eye, and 1 dioptre in the left. The right eje was totelly blind; vision in the left was reduced to $\frac{8}{12}$. The left visual field was normal. The pupils were large and contracted on convergence. The left pupil reacted normally to light; the right pupil reacted consensually with the left, but not on direct illumination. There was slight nystagmus on turning the eyes towards the right side. Hearing in the right ear was slightly diminished, both to aërial and to osseous conduction. The muscles of mastication, face, palate, and tongue were normal. There was no cutaneous ansesthesia or analgesia; nor was there any motor weakness of the trunk or limbs. The gait was unsteady and reeling. There was slight clumsiness in the right upper limb reeling. There was slight clumsiness in the right upper limb on attempted rapid alternate rotation and supination (dysdiadocokinesia). The head was held in the cerebellar attitude, tilted sideways to the left, with rotation of the chin towards
the right. The supinator-jerks, knee jerks, and ankle-jerks were brisk, slightly more so on the right side. There was no ankle clonus, and the plantar reflexes were flexor in type. The sphincters were normal. The cerebro-spinal fluid, which was under increased pressure, contained no abnormal constituents on microscopic examination.

The patient complained of occasional transient blindness in the right eye, and of occasional subjective feelings of numbness in the left face, but without objective anaesthesia on testing.

In view of the intense optic neuritis, the blindness of the right

In view of the intense optic neuritis, the blindness of the right
eye, and the occasional amblyopia of the left, operative intereje, and the occasional amblyo

The cerebellar gait and posture of the head, the slight deafness of the right ear, and the dysdiadocokinesia of the right hand, inclined us to a diagnosis of right-sided extracerebellar growth. Accordingly the right cerebellar fossa was explored, and at the second stage a cystic tumour, the size of a pigeon's egg, was removed from the region of the internal auditory meatus. The patient unfortunately died without regaining consciousness. Post mortem, recent haemorrhage was found all round the pons ind onted by the growth.

CASE III.-Bilateral Extracerebellar Tumours arising from A uditory Nerves. A glass-cutter, 40 years of age noticed gradual deafness in
both ears, more marked in the left ear, of about five years' duration. Twelve months ago he became frequently giddy and staggered in his walk, occasionally falling down. He had occasional occipital head

On examination he had optic neuritis in both eyes, more On examination he had optic neuritis in both eyes, more
intense in the left. The pupils were equal and normal. There intense in the left. The pupils were equal and normal. There was nystagmus on lateral deviation to either side. There was nerve deaness of both ears, more marked in the left ear. He vertigo when he tried to stand or walk; there was no vertigo at rest. The muscles of mastication, the face, palate, and tongue were normal. There was no cutaneous anaesthesia or analgesia. There was moderate dysdisdocokinesia of the left upper limb. The gait was reeling and unstesdy; he did not fall to one side more than the other. The posture of the head was suggestive of cerebellar disease, the chin being elevated were brisk; there was no ankle clonus. The left plantar reflex was extensor in type; the right was doubtful.

The diagnosis made was that of left-sided extracerebellar growth. Antisyphilitic treatment having been negative in its growth. Antisyphilitic treatment having been negative in its
result, it was decided, in view of the advancing optic neuritis, to perform a decomp ressive trephining. This was accordingly done in the left cerebellar region by my colleague, Mr. W. G. Spencer. At the second stage, when the dura mater was opened, the cerebellum bulged and did not pulsate. The cerebellar protrusion rendered exploration of the cranial base difficult. A fortnight later the patient died. At the autopsy there ware two large tumours-one, the size of a hen's egg, in the left. ponto-cerebellar angle; the other, about the size of a cherry,-in the corresponding position on the right side. These tumoure were on the auditory nerves, and compressed the cerebellum without infiltrating $i t$. In addition there were multiple small tumours scattered on the roots of numerous spinal nerves throughout the spine, and one on the spinal accessory nerve on
the left side.

In contrast with these two extracerebellar cases, in both of which we had cochlear or vestibular symptoms, let me now quote an intracerebellar case where there were no symptoms referable to either division of the anditory nerve.

CASE IV.-Right-sided Intracerebellar Tumour.

A girl of 23, who had alwars been thin, began to feel specially exhausted about seven months ago. Four months ago she developed occipital headache and causeless vomiting in the
mornings, which persisted daily for about three weeks. Three mornings, which persisted daily for about three weeks. Three
months ago she consulted an eminent physician, who put her in a nursing home for a month's rest-cure. Six weeks ago this physician discovered optic neuritis, and advised a decom pressive trephining, there being at that time no definite localheadache and the vomiting continued.

On examination the patient, who was of normal intelligence, had marked optic neuritis in both eyes. The visual fields were normal. Smell, taste, and hearing were acute on both sides. The pupils and external ocular movements were normal. There was no nystagmus and no squint. The muscles of mastication, face, palate, and tongue were all normal. There was no motor face, palate, and tongue were all normal. There was no motor
paralysis of the trunk or limbs. There was doubtful unsteadiparalysis of the trunk or limbs. There was doubtful unsteadiress of the right hand on touching the nose. The gait was reeling and unsteady, the patient lurching to the right, and the opposite knee. When standing on one leg she did so less securely on the right foot than on the left. The deep reflexes in upper and lower limbs were normal and equal, and the plantars were flexor in type. There was some tenderness on pressure in the occipital region, more marked on the right side. The reeling gait and the unsteadiness of the right arm and leg pointed to a right-sided cerebellar lesion. Accordingly $\mathrm{Mr}$. C. A. Ballance explored the right cerebellar fossa. At the first stage, on removing the bone, the dura mater bulged with special intensity at a spot about one inch to tho right of the middle (six per minute), and the second stage of the operation had slow (six per minute), and the second stage of the operation had the be done urgently. On opening the dura mater at the site of the local bulge, a jet of fluid escaped from a cystic growth
within the lateral lobe of the cerebellum. The posterior half within the lateral lobe of the cerebellum. The posterior half
of the right lateral lobe, including the growth, was removed. of the right lateral lobe, including the growth, was removed.
The patient made an uninterrupted recovery, although the optic neuritis was somewhat slow in subsiding.

Let us now pass to some examples of syringomyelia or central glioma of the medulla and spinal cord, associated with laryngeal and palatal symptoms.

CASE v.-Syringomyelia with Nuclear Paralysis of the Larynx.

The patient was a married woman of 48 , who complained of tingling pains in the right upper extremity of two years" duration. These pains appeared also in the left upper limb six months ago. Twelve months ago, when having treatment by radiant heat to the right shoulder, the skin was burnt in that region, but produced no pain. Eight months ago her gait months ago she began to have difficulty of swallowing, especiaily with fluids, and had occasional regurgitation. At ithis together with stridor.

On examination, the patient's voice was husky, and there was occasional inspiratory stridor. The optic discs were normal, and there was slight nystagmus on looking to the left. The face, palate, and tongue were normal, also the sterno-mastoids and trapezii. On laryngoscopy, there was bilateral abductor paralysis of the cords. She had an area of dissociated anaesthesia in the head, upper limbs, and upper part of the trunk, excluding the left face. There was no tactile anaesthesia. The upper extremities were normal. The lower muscles were toe when walking. The deep reflexes were normal, and the plantar reflexes were flexor in type. Under treatment by $x$ rays to the cervical region of the spine she improved con. siderably. The lower extremities lost their weakness, and the area of dissociated anaesthesia receded, so that the whole face became normal in sensation.

This was a fairly typical case of syringomyelia, in which the gliomatous process had extended into that part of the vagal nuclei which supplies the laryngeal muscles.

CASE VI.-Syringomyelia with Nuclear Paralysis of Palate,

A lady school teacher, 28 years of age, had noticed for seven years that she had been able to hold her hands closer to the fire than ordinary people, and within the last four years she had frequently burnt herself when ironing linen, not noticing the burns until she saw the blisters. Seven months ago her voice became hoarse, and five months ago she developed inspiratory stridor. Later she had occasional nasal regurgita. tions when swallowing fluids. 
On examination, the voice was particularly husky, with oscasional attacks of inspiratory stridor and of choking, especially when excited. Smell was normal on both sides. The optic dises and visual fields were normal. The pupils reacted normally. There was marked lateral nystagmus in all reacted normally. There was marked lateral nystagmus in all
positions of the eyes. The facial muscles. were normal. positions of the eyes. The facial muscles. Were normal. Hearing was acute. The right side of the palate was paraaimost complete on the right side, slight on the left. The false cords came together well on explosive coughing. The sternomastoids and trapezii were normal. The tongue was wasted, especially on the right side, and showed fibrillary twitchings.

There was no cutaneous anaesthesia, but a characteristic area of therm-anaesthesia and analgesia in the head, secisted ansesthesia was much less intense in the face than elsowhere. In the upper extremities, there was slight wasting of the first dorsal interosseous muscle of the left hand, and the nail of the left thumb was in process of detachment, following a painless whitlow a fortnight previously. The lower limbs were normal in their movements. The deep reflexes were wormal, and the plantars were flexor in type.

This was evidently a case of syringomyelia in which the gliomatous process extended upwards in the medulla to the nuclei of the hypergloseal and vagus, being more marked on the right side.

The next case is a remarkable one of congenital malformation of the medulla and spinal cord, in which wo were fortunate enough to obtain anatomical confirmation of our diagnosis.

CASE VIr.-Congenital Unilateral Intramedullary Lesion of the Medulla.

A man of 41, a hot-potato hawker, came to hospital suffering from chronic bronchitis and chronic nephritis. His voice had always been hoarse. What struck us particularly when he came was the curious deformity of the right side of his neck and jaw. On examination the upper cranial nerves were normal. The right pinna, as you will see from the photograph was much smaller than the left, and the external auditory meatus was narrower and situated higher up than on the left side. The right mastoid process, together with the sternomastoid muscle, was absent. The right side of the mandible was smaller than the left, and he had never had any lower bicuspid or molar teeth on the right side. The facial muscles were normal. The right palate was paralysed. The right vocal cord was fixed in the cadaveric position. The right trapezius, save for a small bundle of its middle fibres, was absent. The right side of the tongue was atrophied, and the omohyoid, stylohyoid, and posterior belly of the digastric were absent, stylohyoid, and posterior belly of the digastric were absent, so also were the depressor muscles of the hyoid bone on the
right side. As a result the common carotid was practically right side. As a result the common carotid was practically its whole extent, and in a photograph could easily be seen its bifurcation into internal and external carotids. There were no sensory or motor abnormalities in the trunk or limbs, and the reflexes, deep and superficial, were normal.

The absence of the right sterno-mastoid and trapezius, toGether with the paralysis of the palate and vocal cord in the same side, led us to diagnose a congenital lesion of the spinal accessory and vagal nuclei on the right side. Further, the atrophy of the right half of the tongue, and the absence of the depressor muscles of the hyoid bone, showed that the lesion extended downwards through the hypoglossal nucleus to the anterior cornu of the upper two or three segments of the spinal anterior cornu of the upper two or three segments of the spinal and the absence of the back teeth with an affection of the and the absence of the back teeth with an affection of the lateral aspect of the medulla and upper segments of the spinal

Some six weeks later the patient died unexpectedly from pontine haemorrhage, and we had an opportunity of verifying our diagnosis. There was absence of the motor nuclei of the vagus, spinal accessory, and hypoglossal on the right side, together with diminution in size of the anterior cornu on the atrophy of the spinal root of the trigeminal nucleus.

Let me now quote two or three examples of acute vascular lesion within the medulla, in which the laryngeal and palatal symptoms gave us the key to the diagnosis.

CASE VIII-Right-sided Thrombosis of the Meduila.

An unmarried lady, aged 63 , who had a chronic mitral regurgitation of many years' standing, developed acute abdominal discomfort during afternoon tea. Some hours later, during sapper, she found a difficulty in swallowing, and a tendency for fluids to regurgitate through the nose. The next morning ner own medical adviser, Dr. McHattie, found the palate severely paralysed. He also noted that the right vocal cord was im. ture and pain on the left side of the body. The urine contained a considerable quantity of albumen.

When I saw her, four days later, there was no unconsciousness, nor any affection of any of the higher cerebral centres. The patient's speech was normal. The articulation was perhaps a patient's speech was normal. The articulation was perhaps a
dittle thick. The optio discs were normal. In connexion with the eyes, there was evidence of paralysis of the oculo-pupillary division of the cervical sympathetic on the right side, there being contraction of the right papil, enophthalmos of the right eye, and slight drooping of the right upper lid without paralysis of the levator. The pupils reacted normally. The only other of the levator. The papils reacted normaly. The only other abnormality in the domain of the cranial nerves was that the
right side of the palate lagged behind the left on phonation, and right side of the palate lagged behind the left on phonation, and
that the right vocal cord moved less freely than the left. There that the right vocal cord moved less freely than the left. There was no cutaneous ansesthesia to the lightest touches. There was, however, total analgesia and therm-anaesthesia on the
left side of the head, trunk, and limbs, except in the territory of the fifth nerve. There was no giddness. The motor furctions of the arms and legs were normal. The knee-jerks were normal and equal, and the plantar reflexes were extensor in type. The temperature was normal. The blood pressure was $164 \mathrm{~mm}$. of in rhythm, and had a systolic mitral bruit.

In this case the total interruption of the paths for temperature and pain on the left side of the body pointed to a lesion on the right side of the pons or medulla close to the surface. The right side of the pons or medula close to the surface. The must be below the middle of the pons. The absence of vertigo must be below the middle of the pons. The absence of vertigo or other anral phenomena, together with the presence of
paralysis of the right palate and vocal cord, pointed to a lesion paralysis of the right

A week later the patient had another attack in which there was superadded a complete right hemiplegia, evidently from a second lesion higher up. This second attack proved fatal in a few hours.

CAsE 1X.-Left-sided Bulbar Thrombosis.

The patjent was a man of 50 , of exceptional intelligence and culture, who, twenty years previously, acquired syphilis in the left eye (by infection from another syphilitic patient who left eye (by infection from another syphilitic patient who coughed in his face). Irido-cyclitis with subsequent blindness followed in the affected eye, in spite of assiduous anti-specific treatment. Ten years ago, when convalescing from an attack and vomiting, and fell persistently to the left. The vertigo lasted a few months, since when it had been intermittent, but after the attack he noticed that he had complete loss of sensibility to pain and temperature in the right side of the body. $\mathrm{He}$ also had at the start some temporary weakness of the left arm and the right leg, which soon cleared up. The left side of the palate and the left vocal cord were completely paraly sed. About three years ago nerve deafness appeare

On examination, the right optic disc and right pupil were normal. There was atrophy of the left optic disc with old normal. There was atrophy of the left optic disc with old choroido-retinitis. The pupil in the blind eye did not react to light, and there was an external strabismus, such as we often observe in a blind eye. There was no true ocular paralysis,
however, nor any nystagmus. Both pupils reacted to light. however, nor any nystagmus. Both pupils reacted to light. eft palate had clesred up, but the left vocal cord was fixed in the cadaveric position. There was no cutaneous anaesthesia, bat there was total analgesia and therm-anaesthesia of the whole of the right side of the body, with the exception of the trigeminal area of the face and anterior half of the scalp. There was no motor weakness or ataxia of the limbs or trunk; the knee-jerks and ankle-jerks were normal. The left plantar reflex was extensor in type, the right was doubtful. There was both delay and precipitancy of micturition. The cerebrospinal fluid contained a large excess of lymphocytes, numbering 28.2 per cur

The eymptoms in this case pointed clearly to a lesion in the left ponto-medullary angle, apparently of the nature of a syphilitic thrombosis of the posterior inferior cerebellar artery, interrupting the path for temperature and pain from the opposite side of the body, and leaving unaffected that for tactile sense, which runs more mesially in the formatio reticalaris. The lesion also implicated the part of the vagal nucleus (nucleus ambiguus) associated with the motor innervation of the palate, which cleared up, and of the vocal cord, which remained paralysed. At the time of onset the adjacent inferior peduncle of the cerebellum was also temporarily implicated, producing the reeling and forced movements to the left, nystagmus, and vertigo, whilst there appears to have been some interference with the pyramidal paths, which largely cleared up.

CASE X.-Left-sided Thrombosis of Medulla. A German waiter, 28 years of age, became violently sick one
morning when drinking a cap of coffee. Two hours later he suddenly felt intensely giddy, saw double, and when he trieduto walk felt himself pulled strongly towards the left side. When I saw him later in the day I found him perfectly intelligent, with normal speech and articulation. The optic discs were normal. There was coarse rotato-lateral nystagmus even with the eyes at rest, not specially exaggerated on looking in various directions. The diplopia had passed off. The external ocular movements were fully executed in all directions. The face, muscles of mastication, and tongue were normal. The left side of the palate was paralysed. The larynx was not examined. There 
weakness or ataxia of the arms and legs as he lay in bed. On asking him to sit up he at once fell to the left. When attempting to walk with support on both sides he also fell to the left. feeling himself forcibly drewn to that side. The knee-jerks and plantar reflexes were normal. The blood pressure was low. plantar refiexes were normal. The blood pressure was low. The above signs pointed to a sudden vascular lesion in the region of the left side of the bulb, implicating the inferior peduncle of the cerebellum and the adjacent nucleus. of the vagus, as evidenced by the forced movements and the unilateral paralysis of the palate.

In a man of his age the likeliest cange was a syphilitic throm bosis. The patient denied all venereal disease, but a loca examination showed the soar of an old chancre, together with a chronic gonorrhoea in fall flood. He was therefore at once placed upon energetic antisyphilitic treatment. Unfortunately I did not see him again.

The last group of cases to which I would direct your attention is that of extramedullary lesions, in which you will note the presence of bulbar symptoms implicating the palate, larynx, and other parts, but without implication of the long sensory or motor tracts of the brain.stem.

CASE XI.-Right-sided Extramedullary Lesion : Caries of Sphenoid Bone.

A man 42 years of age, who denied venereal disease, began to suffer, twelve months before he came under observation, from continuous vertical headache. The pain spread later to the bridge of the nose and to the right infraorbital region. Seven months ago the hearing in the right ear began to be Seven months ago the hearing in the right ear began to be ing, and soon after this his voice beesme uly in swallowing, and soon after this his voice beeame hoarse. Three months ago he not we sujective sensation of numbness together with loss of feeling in the right side of the face, starting below the right eye, and at the same time he observed that his jaw swung to the right when he opened it. Two month generadly feeble and weak' on his legs, but had no localized weakness of any limb.

On examination he was thin and haggard. Mentally, he was intelligent, and his speech was normal. The voice was very hoarse. Smell was acute in both nostrils. Taste as tested with a gentle golvanio current was logt all over tho right gide With a genthe gal of the vertex was heard louder in the right ear; and there was also slight deafness aërially to watch-tick in the right ear. The optic discs and pupils wer normal. There was total paraly sis of the right external rectu with diplopia on looking to the right. The right side of the face and scalp was practically anaesthetic over the right supraorbital region, right upper lip, and right side of nose, in the area of the second division of the flfth. There was wasting an paralysis of the right masseter and temporal muscle, and also of the right external pterygoid, as evidenoed by the swinging of the jaw towards the right side when the mouth was opened. There was slight weakness of the right facial muscles, npper and lower. The right palate and right vocal cord were paralysed. The voice was husky. The patient eould not congh explosively. The right sterno mastoid and right trapering paralyed The right helf of the tong par the moderately wasted aid the righ lide. Thes nor motor weskess of the equal on the two sides. The sphincters were normal. There equal on the two sides. The sphincters were

The above signs pointed clearly to an extramedullary organto lesion involving all the cranial nerves, from the fifth to the lesion involving all the cranial nerves, from the fifth to the

twelfth inolusive, on the

The patient died suddenly from haemoptysis. This we expected to be due to preliminary hremorrhage, but an autopsy showed.that the sphenoid bone was carions and sup parating, and ulceration had occurred in the such vlceration being the immediate cause of the fatel haemorrhage. The brain and membranes were nermel nerve palsies had been prodnced by die nos by the foramine of exit.

CASE XII.-Extramedullary Gumma of Right Side of Medulla.

The patient, a photographer, 29 years of age, acquired syphili seven years ago, for which he underwent two years' treatment. Two months ago he began to have intense frontal headese, and night, without vomiting. Four weeks ago his voice huaky, and he had, some difficulty in swallowing, but no nasal regurgitation.

On examination the optic discs, pupils, external ocular move ments, muscles of mastication, and facial. muscles. were all normal. Smell, tagte, and hearing were acute on both sides. There was total paralysis of the right palate, and the right voosi cord was immobile in the cadaverio position. In addition the right sterno-mastoid and right trapezius were pansigsed and devoid of reaction to faradism. Only a few strands, about the breat th of a pencil, survived in the a few strands, about the breath of a pencil, survived in the trapeziuis-fikres probably
supplied, separately from the spinsl scoessory by the third supplied, separately from the spinsl acoessory, by the thirc and fourth cervical nerves, This wasting of the trapezins cutaneous, and changed the outline of the neok and shopider owing to the outwand displacement of the

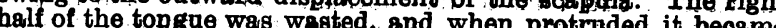
ourved so as to point to the paralysed side. There. Was no cutaneous anaesthesia or analgesia. The motor power of the trunk and limbs was unimpaired. The knee-jerks and anklejerks were normal, and the plantar reflexes were flexor in type. In this case the combination of symptoms pointed to paralysis of the lower roots of the vagus, the spinal accessory, and the of the lower roots of the vagus, the spinal accessory, and the
hypoglossal nerve on the right side. The absence of anaeshypoglossal nerve on the right side. The absence of anaesabsence of vertigo or ataxia, showed that the great conducting absence of vertigo or ataxia, showed that the great conducting with an extramedullary lesion of these three cranial nerves, with an extramedullary lesion
probably syphilitic in origin.

Cases like the above might easily be multiplied, but time does not permit me to detain you longer.

Gentlemen, I have offered you no new facts. Never. theless, I trust that the cases which I have quoted may be of some interest, and that they will serve to exemplify how we may atilize old and familiar facts for the elucidation of less common maladies.

\section{INJURIES TO THE EYE.* \\ BY}

ARCHIBALD STANLEY PERCIVAL, M.B., B.C.CANTAB SENIOR SURGEON TO THE EYE INFIRMARY, NEWCASTLE-UPON-TYNE.

I ONLY propose to speak about a fow of the most common injuries of the eye. If a patient should present himself with one eye that has suddenly become very red, always suspect the presence of a foreign body in it. Let the source of light be on the temporal side, and concentrate it into a cone by means of a large lens on to the eye. If the foreign body is not seen on the cornen, drew down the lower lid and look in the lower fornix; the upper lid should then be everted, and the foreign body will usually be found in the furrow behind the margin of the lid. Finally, it may be situated in the upper fornix. Bodies may lie here un. detected for weeks, as the fornix is never disclosed by merely everting the lid. The everted lid may either be grasped longitudinally with a pair of forceps and rotated again upwards, or a wad of cotton.wool wound round the end of a probe, to form a sort of brush, may be inserted into the fornix, and the whole space wiped out; any particle that lies there will almost certainly be found adhering to the cotton-wool. Incidentally I may say that the easiest way of getting a particle of dust from one's own eye, when lodged in the furrow of the upper lid, is to draw down the upper lid over the lower lid, when the eyelashes of the lower lid will brush out the dust.

The cornea is covered with a layer of epithelium that lies on Bowman's membrane, immediately beneath this is the substantia propria of the cornea. Now, if an abrasion of the cornea occurs which has not perforated Buwman's membrane, no scar will result. If, however, Bowman's membrane be injured, a scar or nebula will certainly result. Injuries with twigs of trees or particles of stone or coal are peculiarly liable to give xise to hypopyon ulcers. In such cases where Bowman's membrane has been injured, I think it well to give drops of atropine and quinine; of this I shall speak presently. It may be noted that abrasions of the cornea with the finger-nail axe especially liable to give rise to recurrent irritation, or traumatic keratalgia, as it is somewhat ponderously named.

When removing a foreign body from the cornea always use a lens. A spring glass like this [shown] is, what I recommend. There is no necessity to fix the eye; the patient, if his eje has been cocainized, will keep his eye quite still, and in most cases the spud will easily lift the foreign body out. Sometimes one will have to use the needle if the particle has got under a flap of the cornea, but in every case I would urge you to use the spring glass. With it difficult cases become easy, and one avoids doing unnecessary damage to the cornea. If the foreign body be very. deeply embedded, or if it protrude through Descomet's : membrane, attempts to displace it with a spud alone will almost certainly push it through into the anterior chamber, and it will probably wound the lens. In such a case a narrow keratome must be introduced into the anterior ohamber near the corneal margin, and it will be found that when its flat : surface is ander the foreign body its removal will be easity effected.

* Read at the all-day scientific meeting of the Northern Braneh of the 